\title{
Zur Diagnostik der Tuberkulose durch inner- liches Einnehmen von Tuberkulin.
}

\author{
Von \\ Dr. med. Tsuneji Sato, \\ Juntendo Hospital, Sakura, Japan.
}

Mit 2 Kurven.

Die Tuberkulindiagnose auf Tuberkulose hat in den letzten Jahren grosse Fortschritte gemacht. Ausser der $\mathrm{K}$ och schen subkutanen Injektion sind die kutane Impfung (von Pirquetsche Allergiereaktion) und die konjunktivale Anwendung (Wolf f-E is ne r sche Konjunktivalreaktion, Calmettesche Ophthalmoreaktion), letztere kurz hintereinander, veröffentlicht worden.

Ir: den letzten Jahren sind auf diesem Gebiete viele Versuche gemacht worden und zahlreiche Arbeiten haben sich damit beschäftigt. Die meisten 'Autoren stimmen darin überein, dass allen drei Methoden eine wichtige Bedeutung für die Erkenntnis des Vorhandenseins eines mit Tuberkulose behafteten Herdes zukommt, insbesondere in früheren Stadien. Andererseits haften aber allen Methoden gewisse Mängel an. Als solche sind folgende Punkte hervorgehoben worden.

\section{Die subkutane Injektion.}

Rechne` man die vor und nach der Injektion zur Beobachtung nötige Zeit hinzu, so hat man für einen Versuch mehr als eine Woche nötig. Ausserdem ist sie sowohl für den Arzt als auch für die Patienten sehr umständlich. Beim positiven Ausfall der Realtion werden, besonders bei empfindlichen Individuen, neben hochgradiger Temperatursteigerung oft unangenehme allgemeine Nebenerscheinungen beobachtet. Auch die lokalen Symptome, die sich durch entzündliche Rötung, Anschwellung und Schmerzhaftig- 
keit kennzeichnen, sind manchmal so stark, dass sie die Patienten mehrere Tage peinigen.

\section{Die kutane Impfung.}

Diese soll im Vergleich zu der obigen ohne nennenswerten Schaden verlaufen, auch wenn die Reaktion positiv ausfällt. Dafür kommt es aber vor, dass die positive Reaktion bei fast gänzlich latenten Tuberkulösen eintritt, die klinisch betrachtet gesund sind.

\section{Die Bindehauteinträufelung.}

Zur Einträufelung des Tuberkulins in das Auge bedarf man keiner besonderen Vorrichtung; sie ist also ganz leicht ausführbar. Aber bei erkrankten Augen kann die Reaktion schwer erkenntlich werden, bei tuberkulöser Erkrankung des Auges werden nicht selten hochgradige Augenentzündungen beobachtet.

Von den drei Proben wird die subkutane Injektion ausschliesslich im Krankenhause gemacht. Die kutane Impfung und die Augeneinträufelung können gelegentlich auch in der Poliklinik gemacht werden, doch ist die ärztliche Untersuchung zu der Zeit, wo die Reaktion erwartet wird, unbedingt notwendig, um festzustellen, ob sie positiv oder negativ verläuft.

Unter Berücksichtigung der hier genannten Punkte lielt ich es für der Mühe wert, zu versuchen, ob das Tuberkulin auch von dem Verdauungskanal resorbiert wird, und ob, wenn das zutrifft, zwischen dem Gesunden und dem mit Tuberkulose behafteten Individuum ein merklicher Unterschied in der Reaktion darauf besteht? Ich plante des weiteren, das innerliche Eimnehmen einer bestimmten Menge Tuberkulin zur Diagnose der Tuberkulose zu benutzen. Nachdem ich meine Versuche begonnen hatte, habe ich aber erfahren, dass ich nicht der erste bin, der auf diesen Plan gekommen ist, dass vielmehr schon Arbeiten in dieser Richtung vorliegen, z. B. Freymuth: Uber Anwendung von Tuberkulinpräparaten per os, Münchner med. Wochenschrift, 1905, S. 62, doch sind dieselben, soviel ich weiss, ziemlich unbeachtet geblieben. Es scheint mir darum nicht unangebracht, meine eigenen Erfahrungen mitzuteilen, obwohl sie. eigentlich noch zu wenig umfangreich sind, als dass man daraus einen endgültigen Schluss ziehen könnte. Soviel kann ich indes mit Bestimmtheit betonen, dass das Tuberkulin-Einnehmen für die Diagnose der Tuberkulose eine grosse Bedeutung hat. Abgesehen davon sind diese Versuche interessant genug, um eine Veröffentlichung zu rechtfertigen. 
3] Zur Diagnostik der Tuberkulose durch innerl. Einnehmen von Tuberkulin. 39

\section{Ausführung.}

Da meine Versuche mit innerlicher Anwendung von Tuberkulin ursprünglich von dem Gedanken ausgegangen ist, damit die Injektion

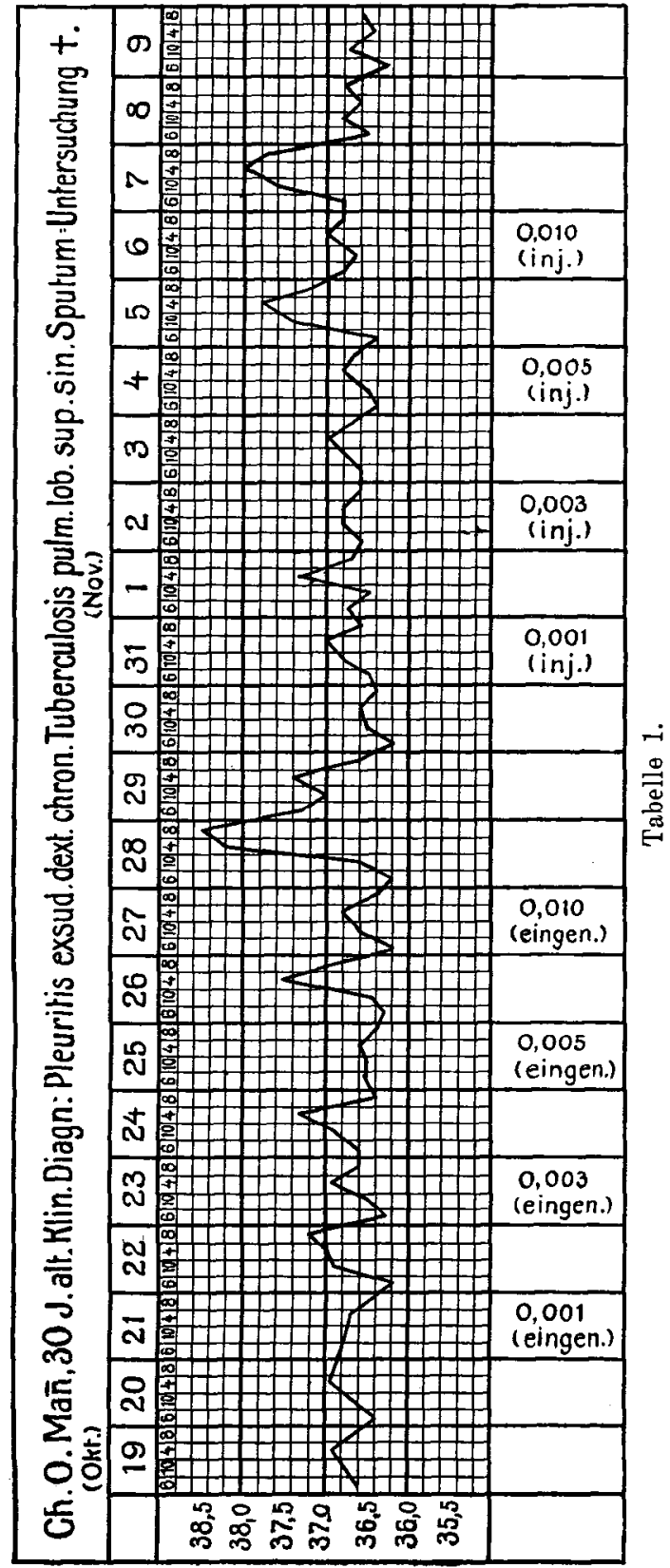


zu ersetzen, so ist ihr Modus im grossen und ganzen der Injektion angepasst worden. Nur habe ich die Dosis etwas grösser als bei der Injektion genommen, weil das per os dargereichte Tuberkulin bei der Passage durch den Verdauungskanal mit den Digestionsflüssigkeiten und dem Magen- und Darminhalt in Berührung kommt und dabei dadurch in seiner Wirksamkeit beeinträchtigt werden kanu.

Vor dem Versuch muss die Temperatur jedes Kranken einige Tage lang täglich viermal gemessen werden. Das Eimehmen lasse ich in den Nachmittagsstunden zwischen 4-5 Uhr vornehmen, da die Reaktion gewöhnlich 15-20 Stunden nachher einzutreten pflegt (somit am Tage), auch deshalb, weil der Magen um diese Zeit zicm-

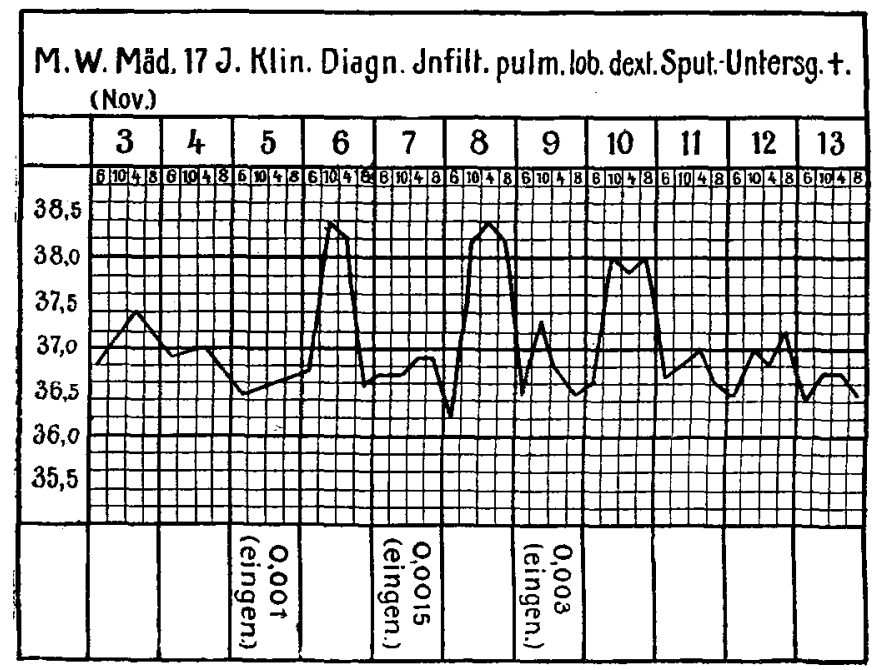

Tabelle 2.

lich leer ist. Ich benutze das Alt-Tuberkulin und beginne mit einer. Dosis von $1 \mathrm{mg}$. Die Lösung wird ror dem jedesmaligen Gebrauch immer new angefertigt und in einer Gelatinekapsel gegeben. Einc Temperatursteigerung im Verlauf des nächsten Tages von mehr als $0,5^{0}$ betrachte ich als positive Reaktion. Zeigt sich auf $1 \mathrm{mg}$ lieine Reaktion, so gebe ich nach drei Tagen $3 \mathrm{mg}$, dann, dasselbe vorausgesetzt, $5 \mathrm{mg}$ und weiter - wie bei der Injektion - bis $z u 10 \mathrm{mg}$. Ist nach dem Einnehmen ron $10 \mathrm{mg}$ Tuberkulin keine verdächtige Temperatursteigerung eingetreten, so betrachte ich das als negativen Ausfall der Probe.

Dem eben beschriebenen Vorgehen gemäss habe ich zuerst die Probe an 12 gesunden Personen (Männer im Alter von 19-54, Frauen von 17-5i Jahren) gemacht, und nach Feststellung des negativen 
Ausfalls der Reaktion bei denselben - d. h. durch den ganzen Zeitraum war keine grössere Temperaturschwankung als $0,3^{0}$ beim Fehlen irgend eines subjektiven Symptoms vorhanden - habe ich weitere Versuche an 10 Phthisikern (darunter bei 8 Sputumuntersuchung: positiv), einem Knaben mit tuberkulöser Peritonitis und 16 nichttuberkulösen Kranken gemacht. Während bei den nichttuberkulösen gar leine Reaktion eintrat, erzielte ich bei den ersteren unter 11 Versuchen 9 positive Reaktionen. Von besonderem Interesse ist das Ergebuis der Injektion nach vorhergegangener innerlicher Darreichung: vori Tuberkulin bei einem Kranken, dessen Temperaturkurve aus der 'Tabelle I ersichtlich ist. Beide Versuche zeigten positive Reaktion.

Die positiv reagierenden Kranken fühlten sich unter der Temperatursteigerung, als hätten sic sich erkältet und klagten über mässige Kopfschmerzen und allgemeine Mattigkeit. Jcdoch dauerte das nur ein paar Stunden. Die nachherige physikalische Untersuchung ergab keine merkbare Teränderung seitens des ursprünglichen Leidens.

Fasse ich nun das Gesagte kurz zusammen, so ergibt sich folgendes:

1. Die innerliche Darreichung von Tuberkulin bedarf keiner besonderen Ubung, ist also für jeden praktischen Arzt äusserst leicht auszuführen.

2. Die bisherige Injektion, Hautimpfung und Augenprobe werden von nervösen Patienten nicht geru geduldet. Besonders bei Kindern ist die Injektion nur schwer ausfülırbar. Das Einnehmen hingegen stösst niemals auf Widerspruch.

3. Dic innerliche Darreichung von Tuberkulin ist nicht nur polikinisch anwendbar, dic Reaktion kann vielmehr auch nach der Selbstbeohachtung des Patienten aus der Temperatursteigerung erkannt werden, olne dass der Arzt den Patienten persönlich zu untersuchen braucht.

4. Bei diesem Vorgehen scheint keine unangenehme Nebenerscheinung vorzukommen. Von Lokalreaktion ist selbstverständlich keine Rede. Dies letztere ist wirklich ein unübertrefflicher Vorzug vor den anderen Methoden.

5. Die Versuche können ohne Mitwissen des Patienten angestellt werden, so dass jede subjektive Beeinflussung ausgeschlossen ist.

6. Man könnte vielleicht einwenden, dass die mehrmalige Temperaturmessung grosse Umstände verursacht. Aber, da Phthisiker oder der Phithisis-Verdächtige gewöhnlich den ärztlichen Befehlen 
gut folgen und sehr aufmerksam auf ihre Erkrankung sind, so wird dio Temperatur im allgemeinen zuverlässig gemessen.

7. Was die Schärfe der Reaktion beim Einnehmen von Tuberkulin betrifft, so ist es notwendig, dass noch weitere Prüfungen an grösserem Material gemacht werden.

8. Bei denjenigen aktiven Phthisikern, bei welchen positive Reaktion eingetreten ist, könnte die innerliche Darreichung von Tuberkulin zu therapeutischem Zwecke versucht werden. 